\title{
Imagerie $X$ de plasma créé par laser
}

\author{
Ph. Troussel, P. Munsch, É. Béchir, M. Pichet, J.Y. Boutin, F. Bridou*, \\ R. Mercier* et J.J. Fermé ${ }^{\star *}$
}

\author{
CEA, Centre d'lle de France, BP. 12, 91680 Bruyères-le-Châtel, France \\ * Institut d'Optique Théorique et Appliquée, Université Paris-Sud, bâtiment 503, BP. 147, \\ 91403 Orsay, France \\ ** Société Européenne de Système Optique, Pôle d'Activité d'Aix-les-Milles, \\ 305 rue Louis Armand, BP. 55000, 13792 Aix-en-Provence, France
}

\begin{abstract}
Résumé : Cet article n'a pas l'ambition d'être une revue exhaustive des diagnostics d'imagerie utilisés pour les plasmas laser, mais plutôt de montrer la variété de l'imagerie basée aujourd'hui, sur l'emploi de microscopes à miroirs ou de lentilles diffractives de Fresnel, la nécessité de les adapter à chaque type de plasma étudié et aussi à la gamme spectrale examinée.
\end{abstract}

\section{INTRODUCTION}

L'irradiation de la matière, par un faisceau laser de grande puissance, conduit à la formation d'un milieu ionisé appelé communément plasma. Pour des éclairements de la matière supérieurs à $10^{13} \mathrm{~W} \mathrm{~cm}{ }^{-2}$, la densité d'énergie injectée dans la cible est telle que la température du plasma dépasse le million de Kelvin. Le maximum de l'émission radiative de ce plasma se situe alors dans le domaine du rayonnement $\mathrm{X}(0,1<\mathrm{h} v<10 \mathrm{keV}$ ou $1 \AA<\lambda<100 \AA)$. L'énergie $\mathrm{X}$ ainsi rayonnée peut atteindre $60 \%$ de l'énergie laser initiale. Il est donc primordial d'étudier ce rayonnement $\mathrm{X}$, car il est le reflet des processus physiques qui assurent le transfert de l'énergie laser à la matière. L'étude de cette émission $\mathrm{X}$ permet de remonter directement aux paramètres fondamentaux du plasma (température, densité, état d'ionisation).

Ces plasmas très denses et très chauds créés par laser (plus simplement appelés plasmas laser) en vue de réaliser la FCI (Fusion par Confinement Inertiel) sont de petite taille (quelques millimètres, voire inférieure au mm) et de courte durée (quelques nanosecondes, voire subpicosecondes). Il y a deux raisons principales pour le développement d'optiques à haute résolution spatiale :

- Les plasmas laser sont le siège de phénomènes souvent indésirables, telles les instabilités, dont les dimensions caractéristiques sont de l'ordre de quelques microns. Pour pouvoir étudier la physique d'implosion (mise en vitesse, symétrisation, instabilités hydrodynamiques) et d'allumage (traceurs, point chaud), on cherchera donc à mettre en évidence des effets fins.

- Le développement des lasers femto-secondes, qui ont une faible divergence et qui permettent de produire des plasmas dont les dimensions sont de l'ordre de $10 \mu \mathrm{m}$, pour lesquels une résolution de quelques microns est donc nécessaire.

Par ailleurs, lorsque l'on désire sonder ces plasmas par un faisceau lumineux, les densités sont telles qu'il est nécessaire d'utiliser un rayonnement $X$.

La résolution désirée est de quelques microns, si possible voisine de $1 \mu \mathrm{m}$, et le grandissement nécessaire se situe donc entre 10 et 50 pour tenir compte de la résolution de 10 à $20 \mathrm{pl} / \mathrm{mm}$ des détecteurs rapides tels que les caméras à balayage de fente (CBFX) ou les tubes obturateurs (TOX). Les optiques doivent selon les applications avoir une résolution spectrale allant de l'eV jusqu'au keV. Elles doivent présenter une grande sensibilité (ouverture) pour bénéficier dans certains cas d'une résolution temporelle.

Pendant de nombreuses années, seule la propriété de propagation en ligne droite des rayons X a été utilisée pour l'imagerie, en particulier avec les chambres à sténopé. Cette technique d'imagerie est d'un emploi simple, largement utilisée, mais présente les inconvénients d'être peu lumineuse et d'avoir une résolution spatiale limitée, soit par la dimension minimale du sténopé (environ $10 \mu \mathrm{m}$ ), lorsque la diffraction n'intervient pas, soit par la diffraction, lorsque le rayonnement observé est trop mou. Il présente l'intérêt de pouvoir l'associer à des détecteurs rapides tels que caméra à balayage de fente (20 ps) ou tubes obturateurs $(80 \mathrm{ps})$. 
Aujourd'hui, les phénomènes de réflexion totale et de diffraction peuvent être mis à profit pour réaliser des optiques. Avec l'augmentation de la puissance des lasers et la recherche d'une meilleure résolution spectrale, on utilise et développe, hormis les chambres citées, trois grands types d'imagerie :

-l'imagerie par réflexion spéculaire : utilisée dans la gamme $100 \mathrm{eV}-10 \mathrm{keV}$; cela recouvre la réflexion totale et la diffraction de Bragg. Les optiques sont constituées par un assemblage de miroirs (ou de cristaux), attaqués, soit sous incidence normale (Schwarschild avec revêtement multicouche), soit sous incidence rasante. Ces optiques étant achromatiques, elles peuvent être non sélectives (miroirs en verre) ou être revêtues de dépôts de couches minces (sélectivité spectrale $\lambda / \delta \lambda 10-100$ ),

-l'imagerie par diffraction : les optiques sont des réseaux de Fresnel, elles sont chromatiques et sont en général couplées à des miroirs multicouches ou cristaux pour avoir une sélectivité spectrale,

- l'imagerie par dispersion : cela concerne les systèmes réalisés à l'aide de cristaux courbes qui permettent d'obtenir typiquement des résolutions spatiales de $10 \mu \mathrm{m}$ avec des résolutions spectrales élevées $(\lambda / \delta \lambda$ 1000).

\section{IMAGERIE PAR RÉFLEXION : MICROSCOPES À MIROIRS}

Lorsque l'énergie laser absorbée par la cible devient importante, la distance entre le plasma et le sténopé doit être augmentée ce qui conduit à une limitation de la résolution spatiale. Cet aspect prend de l'importance avec l'augmentation de la puissance des lasers (LMJ), où il sera difficile de protéger les sténopés. L'avantage principal des optiques à miroirs par rapport aux sténopés est de pouvoir les placer loin de la cible $(>10 \mathrm{~cm})$ tout en conservant la haute résolution spatiale. Pour des énergies faibles (inférieures à $250 \mathrm{eV}$ ), on peut utiliser des miroirs multicouches pour des incidences se rapprochant de la normale avec une réflectivité suffisante (microscope de type Schwarzschild). Dans la gamme $100 \mathrm{eV}$ $10 \mathrm{keV}$ on peut utiliser la réflexion totale sous incidence rasante pour réaliser les microscopes décrits cidessous.

P. Kirkpatrick et A.V.Baez [1] avaient présenté un schéma intéressant de microscope (en abrégé, un KB) comportant deux miroirs sphériques concaves "croisés » (fig.1) permettant d'éliminer l'astigmatisme produit par un miroir concave seul. Utilisé dans plusieurs laboratoires, ce système a permis d'atteindre une résolution de quelques microns, mais dans un champ réduit (champ de rayon qq $100 \mu \mathrm{m}$ ), par suite de l'inclinaison de champ de ce dispositif [2]. Pour s'affranchir de ce défaut, nous avons construit, en collaboration avec l'IOTA, un tel microscope en l'améliorant, par l'utilisation de 4 miroirs au lieu de 2 [3] (KBA). Cette optique s'est avérée être un diagnostic de plasma laser très performant, par son grand champ, «millimétrique», et sa haute résolution (de l'ordre de $7 \mu \mathrm{m}$ dans un champ de $2 \mathrm{~mm}$ ), résolution qui reste encore élevée (meilleure que 25 à $30 \mu \mathrm{m}$ ) dans un champ d'environ $4 \mathrm{~mm}$.

Basée sur l'emploi, d'une part, de miroirs sphériques que l'on sait très bien réaliser avec un excellent état de surface, et d'autre part, de pièces en acier précises, mais usinées de façon courante, sa construction est relativement aisée et son prix raisonnable. Grâce à la faible rugosité des miroirs (5 $\AA$ ), la part du rayonnement diffusé est minime, ce qui permet l'obtention d'images bien contrastées. Les tolérances de fabrication sont sévères. Les 4 miroirs sont issus de la même surface, certains positionnements relatifs des miroirs sont à $1 \mu \mathrm{m}$ et les orientations à 20 '. Cette optique permet un fonctionnement sub-keV à cause de l'énergie de coupure liée aux miroirs en silice $(860 \mathrm{eV})$.

Dans cette perspective, il nous restait à étendre son domaine d'utilisation vers les rayons X plus durs (de 1-10 keV), ce qui peut être obtenu en revêtant les miroirs, soit de métaux très denses comme l'or ou le platine, tout en diminuant l'angle d'attaque, jusqu'à $0,5^{\circ}$ (ce qui semble une limite pratique), soit de multicouches qui, à angle d'attaque constant, réfléchissent un rayonnement plus dur que les dépôts simples. Nous avons donc proposé à l'IOTA d'étudier et de réaliser le même KBA mais avec un dépôt multicouches de W/Si fonctionnant dans une bande d'énergie de l'ordre de $500 \mathrm{eV}$ centrée sur $3,4 \mathrm{keV}$. Ce microscope sera réalisé pour le deuxième semestre 1999. 


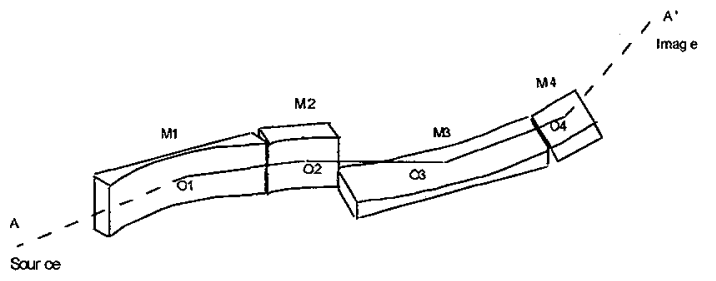

Schéma de principe du KBA

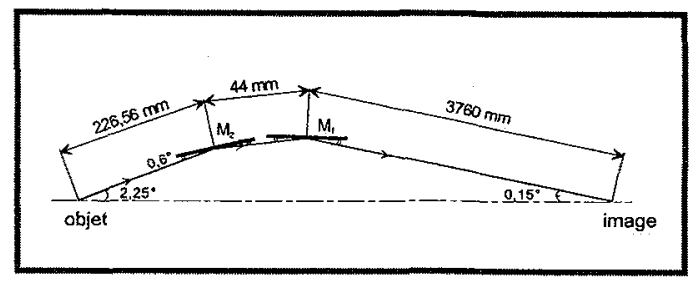

Schéma du Pseudo-Wölter

M. Wölter [4] en 1952 a montré l'intérêt d'associer deux surfaces réflectrices asphériques pour réaliser un système stigmatique pour un point et de plus aplanétique pour le reste du champ. Ce système reste le système le plus performant : son angle solide de collection élevé de $3 \mathrm{msr}$ est 1000 fois plus grand que les microscopes à miroirs classiques. Hormis son coût élevé, la fabrication faisant appel à des techniques de pointe, il présente quelques difficultés de fabrication, et nécessite d'avoir une rugosité de l'état de surface excellente à cause de la symétrie axiale des surfaces asphériques [5].

Nous avons pris l'option de développer avec la société SESO un système proche de celui-ci, le PseudoWölter constitué de deux miroirs à symétrie de révolution et tels que la méridienne des miroirs épousent au mieux la conique dans la partie utile de l'élément. Étant donné la très grande incidence des rayons sur chaque surface $\left(0,6^{\circ}\right)$ les écarts de pente entre l'asphérique et le miroir sont minimisés. Pour obtenir une résolution compétitive avec celle du Wölter, nous avons été amenés à réduire l'ouverture du faisceau à $1 \mathrm{mrad}$, par la dimension de la pupille d'entrée $(200 \mu \mathrm{m})$, limitant la zone utile de chacun des miroirs. Un traitement de surface en nickel sur chaque surface permet d'obtenir une réflectivité d'environ $80 \%$ entre $100 \mathrm{eV}$ et $5 \mathrm{keV}$. Un calcul de réfraction a permis de définir la dimension de la tache image d'un objet et d'évaluer la résolution ultime du système (typiquement $2 \mu \mathrm{m}$ en vertical et $1 \mu \mathrm{m}$ en horizontal). Compte tenu de l'état de surface des miroirs, la résolution expérimentale est comprise entre 3 et $6 \mu \mathrm{m}$ selon la longueur d'onde du rayonnement. Sa faible ouverture numérique lui confère une grande profondeur de champ et il conserve une haute résolution $(5 \mu \mathrm{m})$ dans un champ de $500 \mu \mathrm{m}$ et pour des grandissements variant entre 17 (nominal) et 32. Le Pseudo-Wölter possède son propre système d'alignement. Ce système permet de matérialiser optiquement à mieux que $\pm 50 \mu \mathrm{m}$ la position à laquelle doit être situé l'objet à imager et à mieux que $\pm 750 \mu \mathrm{m}$ la position à laquelle doit être situé le centre du détecteur. En pratique, le système d'alignement projette une mire visible, grâce à une source de forte intensité, simultanément sur la position de l'objet et celle du détecteur. 11 permet ainsi une économie de tirs laser, puisque le diagnostic peut être aligné au préalable avec une source auxiliaire de type générateur $X$.

\section{IMAGERIE PAR DIFFRACTION : LES OPTIQUES DIFFRACTIVES}

Grâce à la maîtrise technologique des couches minces et des micro-technologies, les phénomènes de diffraction sont ainsi mis à profit pour réaliser des optiques focalisantes.

Il s'agit de la diffraction de Fresnel par des réseaux généralement plans, de pas variable. Nous étudions deux types de lentilles pour focaliser le rayonnement $\mathrm{X}$ : soit par transmission normale avec les réseaux à zones de Fresnel circulaires (les lentilles à zones de Fresnel ou LZF), soit en réflexion sous incidence oblique avec des réseaux elliptiques gravés sur des miroirs multicouches (lentilles de Bragg-Fresnel ou LBF). Contrairement aux optiques à miroirs, ces optiques ne possèdent pas d'aberrations géométriques notables, dans notre domaine d'application ; les aberrations de ces lentilles sont principalement chromatiques. Ainsi, les lentilles de Fresnel peuvent être utilisées sans sélectivité avec un rayonnement de raies (plasma d'Aluminium), mais dans le cas plus général on leur associe une sélectivité spectrale typiquement $\lambda / \delta \lambda$ de 1 à 50 . 


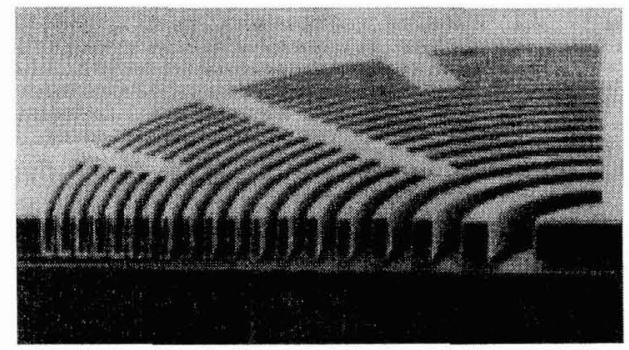

Avec ces lentilles la résolution ultime est limitée par la plus petite largeur de trait que comporte la structure gravée (on obtient actuellement $0,2 \mu \mathrm{m}$ ).

Ces optiques n'ont pas de concurrent pour l'imagerie à haute résolution $(<1 \mu \mathrm{m})$ en dessous de $10 \AA \AA$ de longueur d'onde. La figure ci-contre représente une photo prise au MEB d'une LZF en silicium réalisée par le LETI.

Des tests d'imagerie ont été réalisés au Centre d'études de Limeil-Valenton sur diverses installations. Nos travaux ont montré la faisabilité de réaliser des images de plasmas avec une résolution de quelques microns (typiquement 3-4 $\mu \mathrm{m}$ ) dans le domaine allant de 1 à $2 \mathrm{keV}[6,7]$, à l'aide de deux diagnostics (LZF associée à un miroir multicouche et LBF hors axe [8]). Des expériences comparatives, pour ce domaine spectral, ont montré qu'à ce jour les résolutions spatiales obtenues avec ces deux diagnostics sont voisines, par contre les lentilles hors-axe permettent de séparer les ordres de diffraction et améliorent le contraste. Cette résolution qui est à la pointe de ce que l'on sait faire actuellement, est encore d'un ordre de grandeur plus élevé que la résolution théorique limite $(0,3 \mu \mathrm{m})$, montrant le chemin à parcourir pour l'obtention de telles résolutions auprès d'un plasma laser. L'influence de la dégradation de la résolution due au chromatisme n'est pas encore totalement résolue. Progresser sur ces optiques suppose d'améliorer les technologies dans deux domaines très importants : les traitements multicouches X, et la gravure des réseaux focalisants.

Les lentilles diffractives ont des potentialités en terme de résolutions supérieures aux optiques à miroirs, mais les applications sont spécifiques à chaque longueur d'onde.

\section{CONCLUSION}

Le projet LIL/LMJ fait apparaître un besoin d'imagerie, liée à une spectrométric, avec une haute résolution à la fois spectrale $\left(\lambda / \delta \lambda \approx 10^{3}\right.$ à $\left.10^{4}\right)$ et spatiale $(\mathrm{qq} \mu \mathrm{m})$ dans le but d'imager certaines « espèces » spectrales servant à tracer, soit l'hydrodynamique, soit le mode de combustion de la cible. Nous regardons actuellement la conception et réalisation de dispositifs d'imagerie sur la base des deux types d'optiques citées précédemment, en associant les propriétés de résolution spatiale des lentilles de Fresnel à celles de sélectivité spectrale des cristaux (à la place des miroirs).

\section{Références}

1. Kirkpatrick P., Baez A.V., "Formation of Optical Images by X-Rays", J.O.S.A., 38 (9), Sep. 48, pp. 766-774.

2. Seward F., Dent J., Boyle M., Harper T., et al., "Calibrated "four-color" x-ray microscope for laser plasma diagnostics", Rev. Sci. Instrum. 47(4),vr. 76, pp.464-470.

3. Sauneuf $\mathrm{R}$. et al "Large field high-resolution X-ray microscope for studying laser plasmas". $3412 \mathrm{Rev.}$ Sci. Instrum. 68 (9) Sept 97

4. Wölter $\mathrm{H}$., "Ensembles de miroirs à incidence rasante réalisant des images à l'aide de rayons $X^{\prime \prime}$ (trad. fr.), Annalen der Physik, 10, 1952, pp. 94-114.

5. Aoki and H. Yamaji, Jpn. J. Appl. Phys. 26, 1978 (1987).

6. Thèse de M. Pichet-Thomasset "Imagerie à haute résolution spatiale dans le domaine X-UV à l'aide de lentilles à zones de Fresnel", 2 octobre 1998

7. "Imaging of laser produced plasma at $1.43 \mathrm{keV}$ using Fresnel zone plate and Bragg-Fresnel lens" Rev. Sci. Instrum. à paraître.

8. Thèse de M. G. Cauchon "Imagerie à haute résolution spatiale dans la région 1,5 keV à l'aide de lentilles de Bragg- Fresnel sur miroirs interférentiels multicouches" 18 Septembre 1998. 